\title{
A Novel Riemannian Framework for Shape Analysis of Annotated Surfaces
}

Jiaqi Zaetz

zaetz.2@osu.edu

Sebastian Kurtek

kurtek.1@osu.edu

\author{
Department of Statistics \\ The Ohio State University \\ Columbus, Ohio, USA
}

\begin{abstract}
We present a novel, parameterization-invariant method for shape analysis of annotated surfaces. While the method can handle various types of annotation including color and texture, in this paper we focus on soft landmark annotations. Landmark annotations are commonly provided in various applications including medical imaging where an expert marks points of interest on the objects. Most methods in current literature either study shapes using landmarks only or surfaces only. In either case, the analyst is forced to ignore a lot of useful information. We propose a novel representation of surfaces that can jointly incorporate shape and landmark annotation. Our framework properly removes all shape preserving transformations from the representation space including translation, scale, rotation and re-parameterization. We present results of comparing, averaging and classifying annotated shapes on toy and real data.
\end{abstract}

\section{Introduction}

Shape is a fundamental property of an object and statistical analysis of shapes plays an important role in many applications including handwriting analysis, medical imaging, biometrics, bioinformatics, and many more. Statistical shape analysis was pioneered by D.G. Kendall [15] and Dryden and Mardia [9]. They represented continuous objects (curves, surfaces, etc.) using a finite set of points called landmarks and defined shape as being invariant to rigid motion and scaling. Landmark points are usually chosen by an expert with in-depth knowledge in the application area of interest (anatomical landmarks), and are important in interpreting the subsequent results. However, a major limitation of Kendall's shape analysis is that once the landmarks are chosen the rest of the continuous information is discarded. This results in a large loss of information, which can drastically affect the statistical analysis.

More recently, other representations of 3D objects for shape analysis have become very popular, and many of them have been motivated by medical applications. Studying shapes of $3 \mathrm{D}$ anatomical structures is a promising approach to medical diagnosis and monitoring, because many diseases are linked to alterations of these shapes even before physiological symptoms are present. Several groups have proposed to study shapes of surfaces by embedding them in volumes and deforming these volumes (deformable templates) under the Large Deformation Diffeomorphic Metric Mapping (LDDMM) framework [6, 7, 11, 14, 25]. A closely related approach utilizes inner metrics to describe shape deformations, which are 
prescribed directly on the surface [2]. Other groups study 3D shapes using level sets [23], curvature flows [12], the iterative closest point (ICP) algorithm [1] or the medial axis [3, 10]. The representation of 3D objects by their boundaries, which form parameterized surfaces, also provides a natural framework for statistical shape analysis. Such a representation poses an additional issue of handling the parameterization variability inherent in this type of data. Re-parameterization is the process of determining the correspondence between two or multiple surfaces, and provides information about which point on one surface matches which point on another surface. Some methods $[4,24]$ tackle the problem of re-parameterization by standardization, similar to arc-length in the case of parameterized curves. As was shown in several papers in the case of both curves and surfaces, this type of analysis tends to be very limiting in practice and provides nonintuitive correspondences. A different set of papers treat the re-parameterization step as pre-processing [5, 8]. Once registered, the subsequent statistical analysis of surfaces is performed using standard tools. Because the two steps in these approaches are unrelated, the computed parameterizations tend to be suboptimal, and defining proper parameterization-invariant statistics is not possible. In a series of papers, Kurtek et al. $[18,19,20,21]$ presented a comprehensive framework for parameterization-invariant shape modeling of surfaces based on the $q$-map representation. Later, Jermyn et al. [13] used a different representation of surfaces termed square-root normal fields (SRNFs) for the same purpose, which was based on an elastic Riemannian metric. This method was successfully applied to study shapes of endometrial tissues and cropped faces [16, 17]. The last two approaches overcome the issue of re-parameterization and result in natural shape models.

In many applications such as handwriting analysis and medical imaging, in addition to the continuous surface information, one is often provided with a set of landmark points that have been chosen by an expert. Most of the methods listed in the previous paragraph cannot naturally incorporate this information into the shape analysis framework, and thus ignore this additional information. One exception is recent work of Kurtek et al. [22] where they study surface registration and deformation under few hard landmark constraints. Unfortunately, the assumption of no uncertainty in the landmark placement is very limiting in most applications. It is clear that even an expert practitioner has some level of variability in his or her landmark placement. The current paper extends upon the $q$-map method of Kurtek et al. [18] by defining an extended $q$-map representation of surfaces, which can incorporate additional annotation in the shape analysis framework. While the annotation component can be quite general including color or texture, the current paper studies Gaussian soft landmark annotations and curvature annotations. A curvature annotation can be viewed as an automatic soft landmarking process. We provide results on toy surfaces of revolution and MNIST handwritten digits (represented as graph surfaces) where the gains provided by including landmark annotations are clear. We also study classification of the digits and Attention Deficit Hyperactivity Disorder (ADHD), where the proposed methods give improved performance over methods in current literature that consider the continuous surface information only. Thus, our main contributions are: 1. We define a new representation of annotated surfaces termed the extended $q$-map and define a proper distance between annotated shapes. 2 . We introduce Gaussian soft landmarks that naturally incorporate uncertainty in landmark placement in the shape analysis framework. 3. We introduce automatic soft landmarks via the mean curvature. 4. We define an approximate averaging algorithm for annotated shapes. 5. We present classification results on the MNIST handwritten digits and ADHD medical data. The rest of this paper is organized as follows. Section 2 presents the mathematical framework; Section 3 gives comparison, averaging and classification results for the two types of soft landmark annotations. We close with a brief summary in Section 4 . 


\section{Mathematical Framework}

We present a Riemannian framework for analyzing shapes of annotated, parameterized surfaces. Previous research on parameterization-invariant shape analysis of surfaces has shown that the commonly used $\mathbb{L}^{2}$ metric has important theoretical shortcomings. The main issue is that the action of the re-parameterization group on the space of surfaces is not by isometries. We explain this further next, by considering the case of annotated surfaces, which is the focus of the current paper. Let $S$ denote a $2 \mathrm{D}$ smooth parameterized surface with genus zero, where $D$ is the domain of the surface. In this paper, we are interested in two such domains: unit square $D=[0,1]^{2}$ for quadrilateral surfaces and unit sphere $D=\mathbb{S}^{2}$ for closed (spherical) surfaces. The geometric structure of a surface $S$ can be represented as a smooth embedding of $D$ in $\mathbb{R}^{3}$ denoted by a map $f^{s}: D \rightarrow \mathbb{R}^{3}$. The annotation can be represented by a similar map denoted by $f^{t}: D \rightarrow \mathbb{R}$. Note that, in this work, we only consider 1D functional annotations, but the general framework applies to $n \mathrm{D}$ functional annotations, $n \geq 1$. To study the geometric structure and annotation jointly, the two components are combined into an annotated surface function $f(s)=\left(f^{s}(s) \quad \lambda f^{t}(s)\right)^{T} \in \mathbb{R}^{4}$, where $s=(u, v) \in D$ and the parameter $\lambda \geq 0$ is introduced to control the relative importance of the annotation. When $\lambda$ is small, the target function and statistical analysis puts more weight on the structure component. On the other hand, when $\lambda$ is large, the analysis focuses more on the annotation. The set of all annotated surfaces forms a function space denoted by $\mathcal{F}=\left\{f: D \rightarrow \mathbb{R}^{4} \mid f\right.$ is smooth $\}$. Let $\Gamma$ be the set of all re-parameterizations of the surface domain, $\Gamma=\{\gamma: D \rightarrow D \mid \gamma$ is an orientation-preserving diffeomorphism $\}$. Then, the natural action of $\Gamma$ on $\mathcal{F}$ is by composition, i.e. for an annotated surface $f \in \mathcal{F}$ and a re-parameterization function $\gamma \in \Gamma$, the re-parameterized annotated surface is given by $(f, \gamma)=f \circ \gamma$. It is easy to show that the action of the re-parameterization group on the space of surfaces is not by isometries $\left(f_{1}, f_{2} \in \mathcal{F}, \gamma \in \Gamma\right),\left\|f_{1}-f_{2}\right\| \neq\left\|\left(f_{1}, \gamma\right)-\left(f_{2}, \gamma\right)\right\|$, making the $\mathbb{L}^{2}$ metric inappropriate to use in this context. In fact, equality only holds for reparameterizations $\gamma$ that are volume-preserving (the determinant of the Jacobian of $\gamma$ is 1 at all points), which is very limiting in practice. The proposed framework extends the approach of Kurtek et al. [18] by introducing a generalization of the $q$-map representation of surfaces that allows comparison of annotated shapes using the $\mathbb{L}^{2}$ metric. We present the details next.

In order to study shapes of annotated surfaces, we introduce a novel representation called the extended $q$-map (EQM).

Definition 2.1 Define the extended q-map (EQM) using a mapping $Q: \mathcal{F} \rightarrow \mathbb{L}^{2}$ as $Q(f)=$ $q(s)=\sqrt{|a(s)|} f(s)$, where $a(s)=\frac{\partial}{\partial u} f(s) \wedge \frac{\partial}{\partial v} f(s)$, and $|\cdot|$ is the Euclidean norm in $\mathbb{R}^{6}$.

The wedge product $\wedge$ is a generalization of the cross product to four dimensional vectors, and its norm calculates the directed volume of an area generated by the two vectors. Note that the original $q$-map of Kurtek et al. [18] was defined for surfaces in $\mathbb{R}^{3}$ only using the cross product. Any annotated structure $f \in \mathcal{F}$ can be represented by its EQM $q$. Since $f$ is assumed to be smooth, the space of all EQMs is a subset of $\mathbb{L}^{2}\left(D, \mathbb{R}^{4}\right)$, henceforth simply denoted by $\mathbb{L}^{2}$. Given a re-parameterization $\gamma \in \Gamma$, the EQM of the re-parameterized annotated surface $f \circ \gamma$ is given by $(q, \gamma)=\sqrt{J_{\gamma}}(q \circ \gamma)$, where $J_{\gamma}$ is the determinant of the Jacobian of $\gamma$. Since the space of EQMs is a subset of $\mathbb{L}^{2}$, we equip it with the natural $\mathbb{L}^{2}$ metric to set up a Riemmanian framework. The resulting $\mathbb{L}^{2}$ distance between any two EQMs $q_{1}, q_{2} \in \mathbb{L}^{2}$ is $\left\|q_{1}-q_{2}\right\|=\left(\int_{D}\left|q_{1}(s)-q_{2}(s)\right|^{2} d s\right)^{\frac{1}{2}}$, where $|\cdot|$ is the Euclidean norm in $\mathbb{R}^{4}$, and the geodesic path connecting the EQMs is a straight line. Under this framework, the reparameterization group $\Gamma$ acts on the space of EQMs by isometries. That is, for any $q_{1}, q_{2} \in$ 
$\mathbb{L}^{2}$ and $\gamma \in \Gamma,\left\|q_{1}-q_{2}\right\|=\left\|\left(q_{1}, \gamma\right)-\left(q_{2}, \gamma\right)\right\|$. This allows us to define a parameterizationinvariant framework for shape analysis of annotated surfaces.

Comparison of Annotated Shapes. One must take extra care in accounting for shape preserving transformations of annotated surfaces. In particular, it is important to identify the invariances for each of the two components (structure and annotation) separately and remove them appropriately from the representation space. Shapes of parameterized surfaces are invariant to rigid motion (translation and rotation), scaling, and re-parameterization. Shape analysis can be made invariant to translation and global scaling by normalizing. Invariances to rotation and re-parameterization are dealt with using group actions and equivalence classes. Changing the translation and rotation of the annotation is inappropriate. Thus, the only two invariances we impose on this component are scaling and re-parameterization.

Translation does not change the shape of an object, but it does alter the annotation. Thus, we remove translation variability in the structural component only. The center of mass of the surface can be calculated as $\tilde{v}=\frac{\int_{D} f^{s}(s)\left|a^{s}(s)\right| d s}{\int_{D}\left|a^{s}(s)\right| d s}$, where $a^{s}(s)=\frac{\partial}{\partial u} f^{s}(s) \times \frac{\partial}{\partial v} f^{s}(s)$, and $|\cdot|$ is the Euclidean norm in $\mathbb{R}^{3}$. The centered annotated surface becomes $f(s)=$ $\left(f^{s}(s) \lambda f^{t}(s)\right)^{T}-\left(\begin{array}{ll}\tilde{v} & 0\end{array}\right)^{T}$. We remove the scaling variability, in both the structure and annotation, by normalizing the annotated surface to have unit "volume". The re-scaled annotated surface is $f(s)=\frac{f(s)}{\sqrt{\int_{D}|a(s)| d s}}$. This step re-scales both components using the same constant. If different re-scalings are desired in the analysis, this can be controlled using the parameter $\lambda$. The centered and re-scaled annotated surfaces form the pre-annotated-shape space $\mathcal{C}$. The corresponding EQM pre-annotated-shape space is denoted by $\mathcal{C}_{q}$.

Although rotation does not change the shape of an object, it does change the annotation. Thus, it is desirable to remove the variation due to rotation in the shape component only. To achieve this we define a subgroup of the rotation group $S O(4)$ according to $R=\left(\begin{array}{cc}S O(3) & 0 \\ 0 & 1\end{array}\right) \subset S O(4)$. For any $f \in \mathcal{C}$ and $O \in R, R$ acts on $\mathcal{C}$ according to $(O, f)=O f$. This action is the same on the pre-annotation-shape space of EQMs $((O, q)=O q)$. It is easy to show that the rotation group $R$ acts on $\mathcal{C}_{q}$ by isometries under the $\mathbb{L}^{2}$ metric. Reparameterization changes the mathematical representation of a surface by deforming its coordinate system. However, it preserves the shape and annotation information, and thus, we seek invariance to such transformations for both components. The re-parameterization group $\Gamma$ acts on $\mathcal{C}$ and $\mathcal{C}_{q}$ as stated previously. It follows from the definitions of the rotation and reparameterization groups that their actions on $\mathcal{C}$ and $\mathcal{C}_{q}$ commute, which enables us to define an action of the product group $R \times \Gamma$. To unify all elements of $\mathcal{C}_{q}$ that are within a rotation and/or re-parameterization of each other, we define an equivalence class of an EQM according to $[q]=\{O(q, \gamma) \mid O \in R, \gamma \in \Gamma\}$. Each equivalence class $[q]$ is associated with a unique annotated shape and vice versa. The set of all such equivalence classes $\mathcal{S}_{q}=\left\{[q] \mid q \in \mathcal{C}_{q}\right\}$ is the quotient space $\mathcal{S}_{q}=\mathcal{C}_{q} /(R \times \Gamma)$, and is referred to as the annotated-shape space.

A fundamental goal of shape analysis is to quantify differences between shapes. Since both rotation and re-parameterization act on $\mathcal{C}_{q}$ by isometries under the $\mathbb{L}^{2}$ metric, we can define a distance between any two annotated shapes as a distance between their EQM equivalence classes, i.e. $d\left(\left[q_{1}\right],\left[q_{2}\right]\right)=\min _{O \in R, \gamma \in \Gamma}\left\|q_{1}-\left(O q_{2}, \gamma\right)\right\|$. The computation of this distance requires jointly solving for the optimal rotation $O^{*}$ and re-parameterization $\gamma^{*}$. Searching for the optimal rotation is achieved by Procrustes analysis (singular value decomposition). The search over $\Gamma$ is very similar to the problem considered in $[18,20]$ and we utilize their algorithm for this purpose. We refer the reader to their papers for details.

Approximate Averaging of Annotated Shapes. A fundamental goal of statistical shape 
analysis is to compute an average shape. This can be accomplished using the Karcher mean in $\mathcal{S}_{q}$ as follows. Given a set of normalized annotated surfaces $\left\{f_{1}, \ldots, f_{n}\right\} \in \mathcal{C}$ represented using their EQMs $\left\{q_{1}, \ldots, q_{n}\right\} \in \mathcal{C}_{q}$, the annotated shape Karcher mean is given by $[\bar{q}]=$ $\operatorname{argmin}_{[q] \in \mathcal{S}_{q}} \sum_{i=1}^{n} d\left([q],\left[q_{i}\right]\right)^{2}$ (a gradient descent algorithm to compute it is presented in [9]). The resulting average is an equivalence class of annotated surfaces, and thus, we select one representative: $\bar{q} \in[\bar{q}]$. Unfortunately, in order to visualize the average annotated shape, one must apply the inverse mapping $Q^{-1}$ to $\bar{q}$ to obtain the corresponding annotated surface. But, this mapping is not easy to compute. Thus, instead of computing the exact average annotated shape, we resort to an approximate algorithm, which does not require this step. We outline the algorithm next. First, initialize with an approximate average annotated shape by computing $\bar{f}_{0}=\frac{1}{n} \sum_{i=1}^{n} f_{i}$ and normalizing it with respect to translation and scaling. Note that we are now using the original surfaces rather than their EQMs. Also, compute the EQM representations $\left\{q_{i}\right\}$ of $\left\{f_{i}\right\}$ for $i=1, \ldots, n$, and the EQM $\bar{q}_{0}$ of $\bar{f}_{0}$. Then, iteration $j$ of the algorithm proceeds as follows. First, find the optimal rotation and re-parameterization of each $q_{i}$ with respect to the current estimate of the average annotated shape $\bar{q}_{j}$ using $\left(O_{i}^{*}, \gamma_{i}^{*}\right)=$ $\arg \min _{O \in R, \gamma \in \Gamma}\left\|\bar{q}_{j}-\left(O q_{i}, \gamma\right)\right\|$. Apply these transformations to each of the corresponding surfaces, $f_{i}^{*}=O_{i}^{*}\left(f_{i} \circ \gamma_{i}^{*}\right)$, and compute the perturbation update using $\bar{w}=\frac{1}{n} \sum_{i=1}^{n}\left(f_{i}^{*}-\bar{f}_{j}\right)$. Then, update the current estimate of the average annotated shape using $\bar{f}_{j+1} \stackrel{n}{=} \bar{f}_{j}+\varepsilon \bar{v}$, where $\varepsilon>0$ is a small step size. Repeat this procedure until convergence and at the last step, normalize the final average annotated shape with respect to translation and scaling.

\section{Experimental Results Using EQMs}

In this section, we present experimental results of comparing and averaging annotated shapes with two different types of annotation. The first type considers the inclusion of soft landmark constraints into shape analysis of surfaces. The term "soft landmark" refers to a point of interest with small additional uncertainty in its placement on the surface. The main drawback of the soft landmark annotation is that it is determined manually, thus requiring a lot of the expert's time and resources. Thus, as a second type of annotation, we consider the mean curvature of the surface. We choose to work with the mean curvature rather than the Gaussian curvature due to its lower susceptibility to noise. A curvature annotation can be viewed as an automatic soft landmarking process where points with high and low curvature are forced into correspondence (mathematical landmarks). We present results on toy surfaces of revolution, images of handwritten digits from the MNIST database viewed as graph surfaces, and subcortical structures for classification of Attention Deficit Hyperactivity Disorder (ADHD). We assess the matching/comparison results by displaying the linear interpolation path between the two registered surfaces: $F(t)=(1-t) f_{1}+t\left(O^{*}\left(f_{2} \circ \gamma^{*}\right)\right), 0 \leq t \leq 1$. The quality of annotated shape comparisons is closely related to the nature of the linear interpolation.

Soft Landmark Annotation. We represent the soft landmark annotation as a mixture of equally weighted bivariate Gaussian distributions centered at the landmark locations with uncertainty contained in the covariance. A soft landmark annotation ensures not only the matching of the overall geometric information of the surfaces, but also forces correspondence between the selected points. Let $s_{1}, s_{2}, \ldots, s_{k} \in D$ denote the coordinates of the selected landmark points. For each provided landmark, we place a bivariate Gaussian distribution centered at the landmark location. Thus, for $s_{i} \in D, f_{i}^{t}(s)=\frac{1}{2 \pi \sqrt{|\Sigma|}} e^{-\frac{1}{2}\left(s-s_{i}\right)^{T} \Sigma^{-1}\left(s-s_{i}\right)}$, 


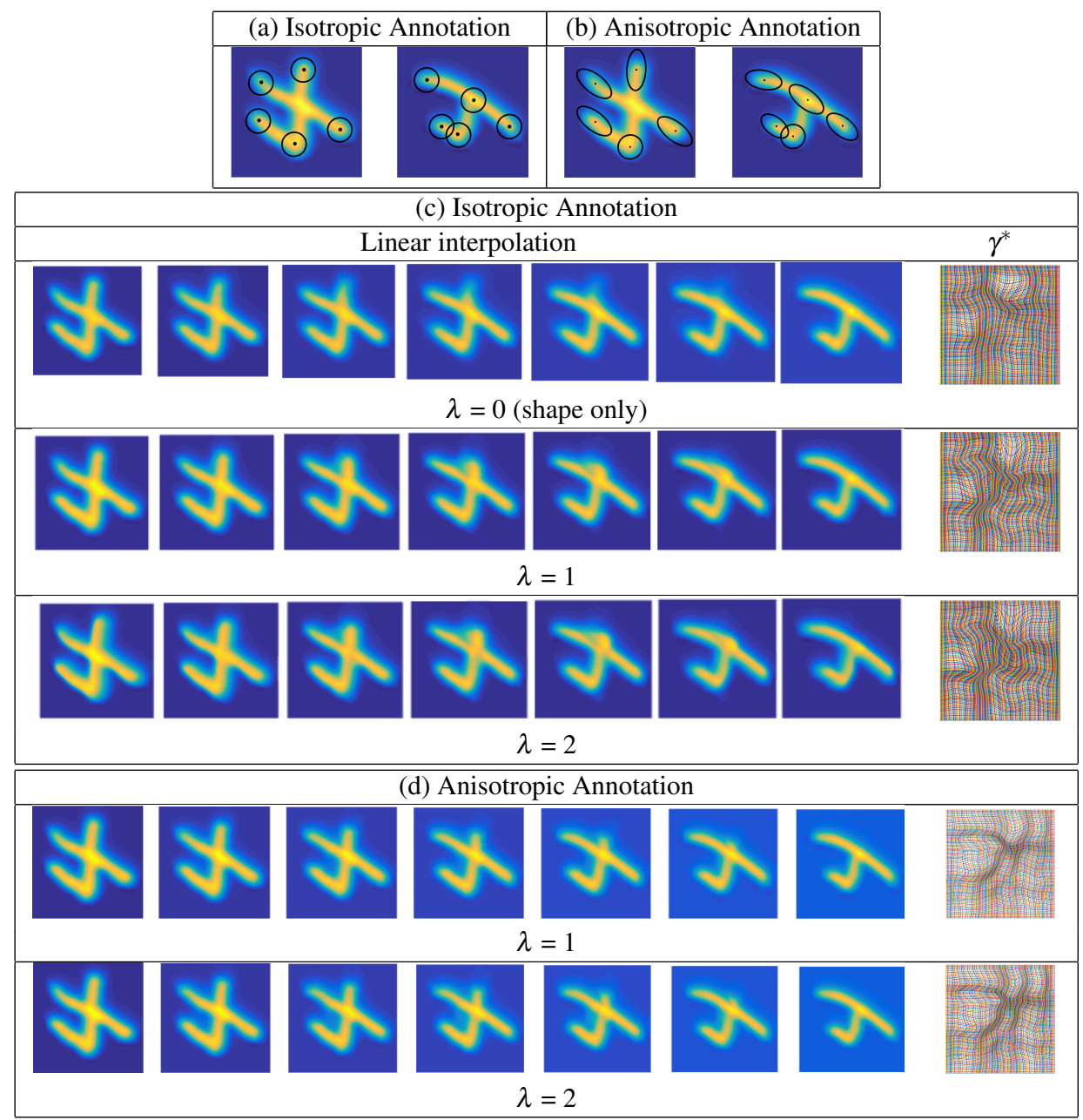

Figure 1: Isotropic (a) and anisotropic (b) Gaussian landmark annotations of two graph surfaces of the digit ' 4 '. Linear interpolation between the two annotated shapes and the optimal re-parameterizations $\gamma^{*}$ of the target surface for isotropic (c) and anisotropic (d) Gaussian landmark annotations (the source image is on the leftmost and the target image is on the rightmost). Note: when $\lambda=0$ the deformation is the same for both cases.

where $\Sigma=\left(\begin{array}{cc}\sigma_{1}^{2} & \sigma_{12} \\ \sigma_{12} & \sigma_{2}^{2}\end{array}\right)$ is the covariance matrix, and $|\Sigma|$ is its determinant. The confidence parameters $\sigma_{1}$ and $\sigma_{2}$ control the coverage of the soft landmarks, while the parameter $\sigma_{12}$ controls the orientation. A smaller $\sigma_{1} / \sigma_{2}$ decreases the coverage of the soft landmarks, and thus, restricts the matching to a smaller neighborhood around the landmark point. The parameter $\lambda$ controls the overall importance of the soft landmarks in the comparison. The full annotation is defined as an equally weighted mixture of the $k$ Gaussians: $f^{t}=\frac{1}{k} \sum_{i=1}^{k} f_{i}^{t}$.

Figure 1 shows two graph surfaces of the digit ' 4 '. In panel (a), we annotated the two surfaces using five isotropic Gaussian landmarks reflecting equal uncertainty in their placement in all directions. In panel (b), we annotated the same two surfaces using five anisotropic 
Gaussian landmarks centered at the same locations but with greater uncertainty along the handwritten strokes (this type of annotation is much more intuitive). The annotations are displayed on the digits using ellipses. The main difference between these two digits lies in the ending point of the ' $L$ ' shaped stroke. It crosses over the straight stroke on the first digit, and ends exactly on the straight stroke on the second digit. This type of variation reflects natural differences in writing styles and must be accounted for in the analysis. Figure 1(c) shows the comparison results for the two digits when using the isotropic Gaussian annotation with a fixed $\sigma_{1}=\sigma_{2}=0.05$ and $\lambda=0,1,2$. Note that when $\lambda=0$, only the structural component is used for comparison. In this case, the part of the ' $\mathrm{L}$ ' shaped stroke that crosses over the straight stroke on the first digit fades away during the deformation. This is not a natural deformation between the two digits. In particular, one would expect the additional part of the ' $L$ ' shaped stroke to contract toward the straight stroke. When we increase $\lambda$ to 1 or 2, the soft landmark annotation starts playing a role and we see a different behavior. The additional part of the ' $\mathrm{L}$ ' shaped stroke begins to shrink toward the cross point, but the folding motion toward the straight stroke is not very natural. Thus, we try to improve this result using anisotropic Gaussian soft landmarks. These results are presented in panel (d) for $\lambda=1,2$. In both cases, we see a much improved deformation. By changing the structure of the landmark uncertainty we are able to generate a natural deformation between the two digits where the extra part of the ' $L$ ' shaped stroke nicely contracts toward the straight stroke. This is the type of handwriting variation that is expected. Figure 2 displays deformation results for two graph surfaces of a digit ' 6 '. Both surfaces were annotated with isotropic soft landmarks (we do not display the annotation for brevity). This case is challenging due to the missing part along the loop of the second digit. We display the comparison results for $\sigma_{1}=\sigma_{2}=0.05$ and $\lambda=0,1,3$. Again, when no soft landmark annotation is used the missing part uniformly appears along the path. A more natural deformation would involve the left part of the loop on the second digit to wrap around and close the right part of the loop. As we increase the parameter $\lambda$, we in fact observe such a deformation. Thus, the soft landmarks play an important role in the matching and comparison of digit graph surfaces.

As a final experiment involving soft landmark annotations, we performed a small classification experiment on a subset of 100 handwritten digit graph surfaces (10 examples of each digit) from the MNIST database. Performance was measured using leave-one-out nearest neighbor classification based on the annotated shape distance. The digit surfaces were annotated using isotropic landmarks and the results were computed for varying values of $\lambda$. The results are presented in Table 1. The final column presents the result of a state-of-the-art method for shape analysis of surfaces (without annotation). Note that when $\lambda=0.75$, giving some influence to the soft landmark annotation, the classification accuracy increases from $81 \%$ (when $\lambda=0$ ) to $99 \%$. The method of Jermyn et al. [13] achieves a $91 \%$ classification rate. Thus, the proposed method outperforms the state-of-the-art in this classification experiment. While in this evaluation we computed the classification result for various types of $\lambda$, one can learn an optimal value of this parameter through cross-validation.

Curvature Annotation. The curvature of a surface can be used as an automatic landmarking procedure where areas of high curvature (in absolute value) are forced to match. Figure 3 presents three comparison results for surfaces of revolution with a mean curvature annotation. In each example, we display the deformation between the two surfaces when $\lambda=0$ and with $\lambda>0$ where the annotation plays a significant role. In all examples, we notice a striking improvement in the matching/comparison achieved by including the curvature annotation.

We also report preliminary classification results for an ADHD study. In this data there was a total of 34 subjects with 19 patients and 15 controls. This dataset was previously 


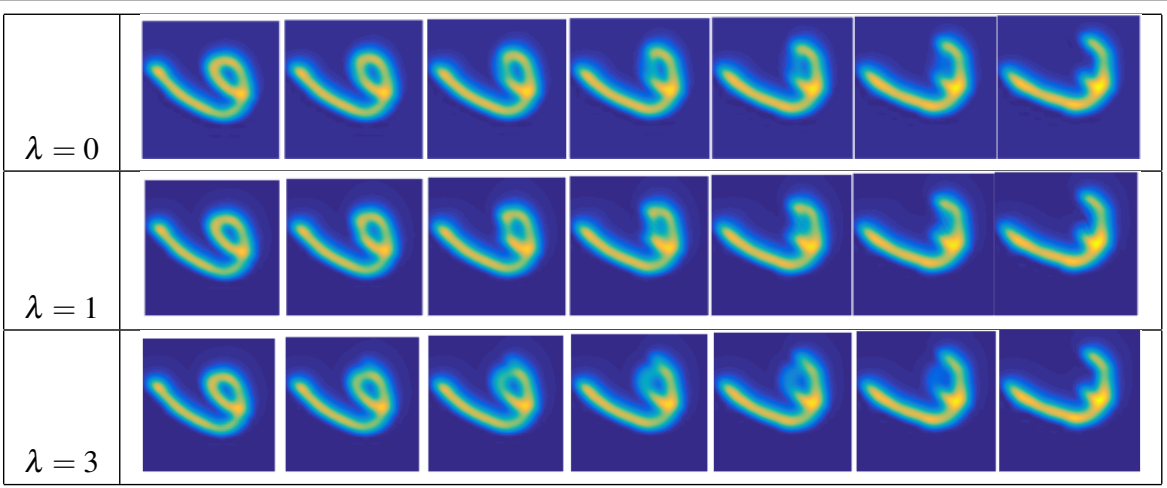

Figure 2: Linear interpolation between two '6' digits with an isotropic Gaussian annotation. The source image is on the leftmost and the target image is on the rightmost.

\begin{tabular}{|c|c|c|c|c|c|c|c||c|}
\hline$\lambda$ & 0 & 0.25 & 0.5 & 0.75 & 1 & 1.25 & 1.5 & {$[13]$} \\
\hline \hline CR (\%) & 81 & 92 & 98 & $\mathbf{9 9}$ & 97 & 94 & 92 & 91 \\
\hline
\end{tabular}

Table 1: Leave-one-out nearest neighbor classification rate (CR) for the proposed method with varying values of $\lambda$ and the method in [13].

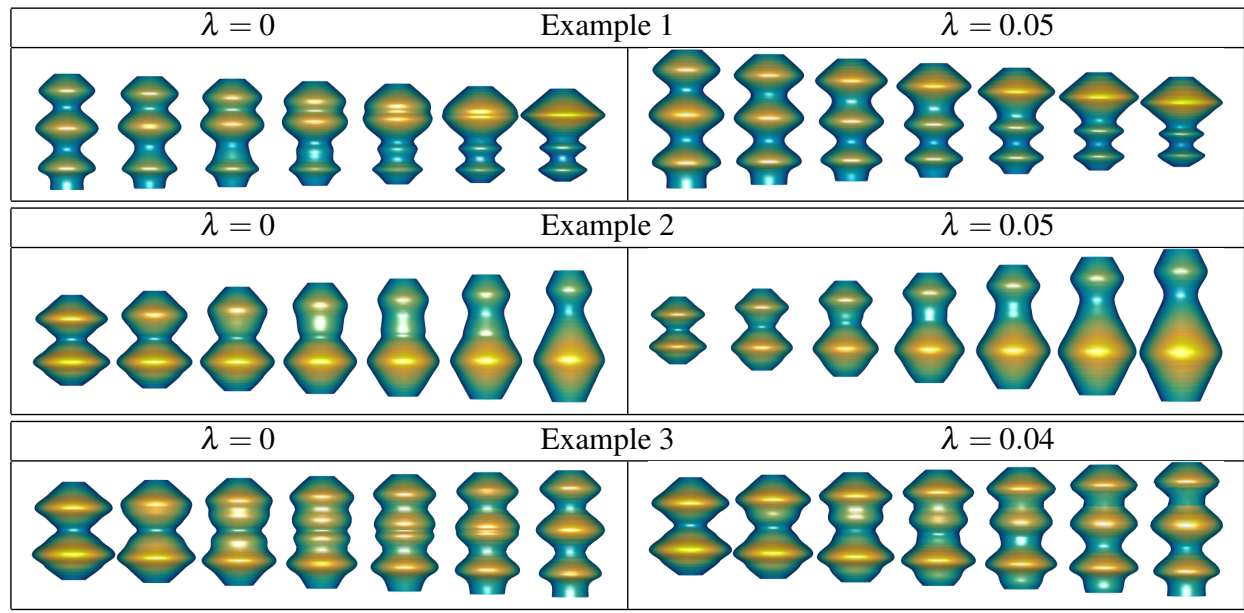

Figure 3: Three examples of deformations between surfaces of revolution with a mean curvature annotation. In each case, we compare the shape only deformation $(\lambda=0$, left) to the annotated shape deformation $(\lambda>0$, right $)$.

analyzed in $[19,20]$; please refer to those papers for a detailed description. In this paper, we study the performance of the proposed framework by computing the leave-one-out nearest neighbor classification rate based on three different subcortical structures annotated using their curvature (Figure 4) for varying values of $\lambda$. The results are presented in Table 2. It is evident from the results that including the curvature annotation in this problem can yield improved classification performance over the shape only case [18]. For example, in the case of the left caudate, when $\lambda$ is increased to 0.25 , the ADHD classification accuracy improves 


\begin{tabular}{|c|c|c|}
\hline Left Caudate & Left Putamen & Left Thalamus \\
\hline & &
\end{tabular}

Figure 4: Structures used for ADHD classification. In each panel, we show the structural component (left), the curvature on a unit sphere (middle) and the annotated surface (right).

\begin{tabular}{|c|c|c|c|c|c|c|c|}
\hline & \multicolumn{7}{|c|}{ Classification Rate (\%) } \\
\hline Structure $\backslash \lambda$ & $0[18]$ & 0.25 & 0.5 & 0.75 & 1 & 1.5 & 2 \\
\hline \hline L. Putamen & 82.4 & 79.4 & 79.4 & 82.4 & $\mathbf{8 5 . 3}$ & 82.4 & 70.6 \\
\hline L. Caudate & 47.1 & $\mathbf{5 5 . 9}$ & 52.9 & $\mathbf{5 5 . 9}$ & 52.9 & 44.1 & 47.1 \\
\hline L. Thalumus & 61.8 & $\mathbf{6 4 . 7}$ & 61.8 & 55.9 & 58.8 & 50.0 & 47.1 \\
\hline
\end{tabular}

Table 2: ADHD classification using three left subcortical structures.

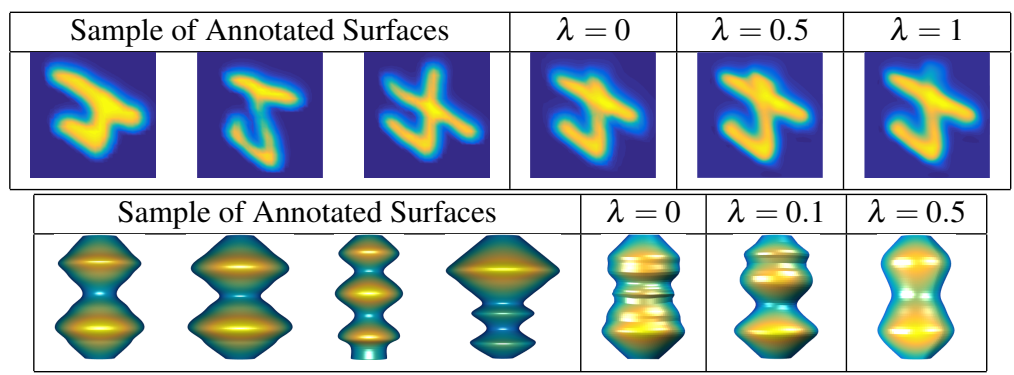

Figure 5: Averaging results for three handwritten digits with Gaussian soft landmark annotation (top) and four surfaces of revolution with a curvature annotation (bottom).

by almost $10 \%$. In future work, we plan to explore this problem in more depth by considering other subcortical structures and even ensembles of multiple structures.

Averaging of Annotated Shapes. The final set of results shown in Figure 5 considers averaging of annotated shapes using the algorithm outlined in Section 2. In the first example, we consider three graph surfaces of the digit '4' annotated using isotropic Gaussian landmarks. When the annotation is included in the analysis, the average shapes preserve more geometric features from the given data. In the second example, we consider four curvature annotated surfaces of revolution with different numbers of peaks and valleys. Here, as $\lambda$ increases, the average shape contains fewer peaks and becomes smoother.

\section{Summary}

We defined a Riemannian framework for shape analysis of annotated surfaces. We represent the annotated surface using an extended $q$-map transformation to compare and average shapes in the presence of soft landmarks. We have applied this framework to classification of handwritten digits and ADHD, and have shown improved performance over previous methods. In future work, we would like to consider other types of annotation including color and texture. We would also like to study variability in annotated shapes using principal component analysis and utilize Gaussian likelihoods for improved classification. 


\section{References}

[1] A. Almhdie, C. Léger, M. Deriche, and R. Lédée. 3D registration using a new implementation of the ICP algorithm based on a comprehensive lookup matrix: Application to medical imaging. Pattern Recognition Letters, 28(12):1523-1533, 2007.

[2] M. Bauer and M. Bruveris. A new Riemannian setting for surface registration. In Proceedings of MICCAI Workshop on Mathematical Foundations of Computational Anatomy, pages 182-193, 2011.

[3] S. Bouix, J.C. Pruessner, D.L. Collins, and K. Siddiqi. Hippocampal shape analysis using medial surfaces. NEUROIMAGE, 25:1077-1089, 2001.

[4] C. Brechbühler, G. Gerig, and O. Kübler. Parameterization of closed surfaces for 3D shape description. Computer Vision and Image Understanding, 61(2):154-170, 1995.

[5] J. Cates, M. Meyer, P.T. Fletcher, and R. Whitaker. Entropy-based particle systems for shape correspondence. In Proceedings of MICCAI Workshop on Mathematical Foundations of Computational Anatomy, pages 90-99, 2006.

[6] J.G. Csernansky, L. Wang, D.J. Jones, D. Rastogi-Cru, G. Posener, J.A. Heydebrand, J.P. Miller, U. Grenander, and M.I. Miller. Hippocampal deformities in schizophrenia characterized by high dimensional brain mapping. American Journal of Psychiatry, 159:1-7, 2002.

[7] C. Davatzikos, M. Vaillant, S. Resnick, J.L. Prince, S. Letovsky, and R.N. Bryan. A computerized method for morphological analysis of the corpus callosum. Journal of Computer Assisted Tomography, 20:88-97, 1996.

[8] R.H. Davies, C.J. Twining, T.F. Cootes, and C.J. Taylor. Building 3-D statistical shape models by direct optimization. IEEE Transactions on Medical Imaging, 29(4):961$981,2010$.

[9] I.L. Dryden and K.V. Mardia. Statistical Shape Analysis. John Wiley \& Son, 1998.

[10] K. Gorczowski, M. Styner, J.Y. Jeong, J.S. Marron, J. Piven, H.C. Hazlett, S.M. Pizer, and G. Gerig. Multi-object analysis of volume, pose, and shape using statistical discrimination. IEEE Transactions on Pattern Analysis and Machine Intelligence, 32(4): 652-666, 2010.

[11] U. Grenander and M.I. Miller. Computational anatomy: An emerging discipline. Quarterly of Applied Mathematics, LVI(4):617-694, 1998.

[12] X. Gu, S. Wang, J. Kim, Y. Zeng, Y. Wang, H. Qin, and D. Samaras. Ricci flow for 3D shape analysis. In Proceedings of International Conference on Computer Vision, 2007.

[13] I.H. Jermyn, S. Kurtek, E. Klassen, and A. Srivastava. Elastic shape matching of parameterized surfaces using square root normal fields. In Proceedings of European Conference on Computer Vision, pages 804-817, 2012.

[14] S.C. Joshi, M.I. Miller, and U. Grenander. On the geometry and shape of brain submanifolds. Pattern Recognition and Artificial Intelligence, 11:1317-1343, 1997. 
[15] D.G. Kendall. Shape manifolds, procrustean metrics and complex projective spaces. Bulletin of London Mathematical Society, 16:81-121, 1984.

[16] S. Kurtek and H. Drira. A comprehensive statistical framework for elastic shape analysis of 3d faces. Computers \& Graphics, 51(0):52 - 59, 2015.

[17] S. Kurtek, Q. Xie, C. Samir, and M. Canis. Statistical model for simulation of deformable elastic endometrial tissue shapes. Neurocomputing. In Press.

[18] S. Kurtek, E. Klassen, Z. Ding, and A. Srivastava. A novel Riemannian framework for shape analysis of 3D objects. In Proceedings of IEEE Conference on Computer Vision and Pattern Recognition, pages 1625-1632, 2010.

[19] S. Kurtek, E. Klassen, Z. Ding, M.J. Avison, and A. Srivastava. Parameterizationinvariant shape statistics and probabilistic classification of anatomical surfaces. In Proceedings of Information Processing in Medical Imaging, 2011.

[20] S. Kurtek, E. Klassen, Z. Ding, S.W. Jacobson, J.L. Jacobson, M.J. Avison, and A. Srivastava. Parameterization-invariant shape comparisons of anatomical surfaces. IEEE Transactions on Medical Imaging, 30(3):849-858, 2011.

[21] S. Kurtek, E. Klassen, J.C. Gore, Z. Ding, and A. Srivastava. Elastic geodesic paths in shape space of parameterized surfaces. IEEE Transactions on Pattern Analysis and Machine Intelligence, 34(9):1717-1730, 2012.

[22] S. Kurtek, A. Srivastava, E. Klassen, and H. Laga. Landmark-guided elastic shape analysis of spherically-parameterized surfaces. Computer Graphics Forum (Proceedings of Eurographics 2013), 32(2):429-438, 2013.

[23] R. Malladi, J.A. Sethian, and B.C. Vemuri. A fast level set based algorithm for topology-independent shape modeling. Journal of Mathematical Imaging and Vision, 6:269-290, 1996.

[24] M. Styner, I. Oguz, S. Xu, C. Brechbuhler, D. Pantazis, J. Levitt, M.E. Shenton, and G. Gerig. Framework for the statistical shape analysis of brain structures using SPHARM-PDM. In Proceedings of MICCAI Open Science Workshop, 2006.

[25] M. Vaillant and J. Glaunes. Surface matching via currents. In Proccedings of Information Processing in Medical Imaging, pages 381-392, 2005. 